\title{
Um Quadro de Análise do Conhecimento Estatístico para Ensinar de Futuros Professores ${ }^{1}$
}

\author{
Hélia Oliveira \\ Instituto de Educação, Universidade de Lisboa \\ hmoliveira@ie.ulisboa.pt \\ Ana Henriques \\ Instituto de Educação, Universidade de Lisboa \\ achenriques@ie.ulisboa.pt
}

\begin{abstract}
Resumo
Neste estudo debruçamo-nos sobre o conhecimento para ensinar investigações estatísticas de futuros professores do $3 .^{\circ}$ ciclo do ensino básico e do ensino secundário, tendo por base um quadro teórico que articula dois domínios: o conhecimento estatístico para ensinar e o pensamento estatístico. Os resultados mostram: i) a pertinência do quadro teórico adotado, podendo constituir uma referência para a construção de situações a explorar na Didática da Estatística, e para colocar em evidência aspetos importantes do conhecimento estatístico para ensinar que os futuros professores estão a desenvolver; e ii) a potencialidade da discussão de episódios de sala de aula, na formação inicial de professores.
\end{abstract}

Palavras-chave: Conhecimento estatístico para ensinar; Formação inicial de professores; Investigações estatísticas; Pensamento estatístico.

\section{A Framework for Analysing Prospective Teachers' Statistical Knowledge for Teaching}

\begin{abstract}
In this study we analyse prospective teachers' knowledge for teaching statistical investigations, for middle and secondary schools, relying on a framework that combines two domains: statistical knowledge for teaching and statistical thinking. The results show: i) that the adopted framework may be used as a reference for the elaboration of didactical situations in teacher education and for encouraging the emergence of important features of the statistical knowledge for teaching that prospective teachers are developing; and ii) the potential of promoting the discussion of classroom episodes in initial teacher education settings.
\end{abstract}

Keywords: Statistical knowledge for teaching; Initial teacher education; Statistical investigations; Statistical thinking.

\footnotetext{
${ }^{1}$ Boletim Gepem (Online) ISSN: 2176-2988 | n. 64 - Jan./Jun. 2014 (texto em diagramação).
} 


\section{Introdução}

Os currículos de diversos países têm vindo a apresentar mudanças no que concerne ao ensino da Estatística, reforçando o seu lugar na formação dos alunos (CAMPOS; CARZOLA; KATAOKA, 2011; NEWTON; DIETIKER; HORVATH, 2011) e estabelecendo objetivos mais ambiciosos que visam o desenvolvimento do seu pensamento estatístico (FRANKLIN et al., 2005). Assumindo uma perspectiva mais holística, destaca-se a importância de familiarizar os alunos com aspetos centrais do método estatístico, como a formulação de questões ou o planeamento e recolha de dados que, sendo mais complexos, podem constituir um grande desafio para os professores (NEWTON; DIETIKER; HORVATH, 2011). Em particular, vários estudos têm revelado as dificuldades dos professores em desenvolver investigações estatísticas com os alunos, reforçando a necessidade de dar atenção a esta atividade na formação inicial, de modo a desenvolver uma compreensão do próprio processo estatístico e um conhecimento estatístico para ensinar que promova o pensamento estatístico dos alunos (BURGESS, 2009; HEATON; MICKELSON, 2002).

Reconhecendo a importância de promover o conhecimento do professor relativo ao ensino de investigações estatísticas, desenvolvemos uma proposta que contempla esse domínio, numa disciplina de Didática da Matemática de um curso de formação inicial de professores de Matemática. Neste estudo, em que adotamos o quadro de Burgess (2009), analisamos os aspetos do conhecimento estatístico para ensinar investigações que os futuros professores de $3 .^{\circ}$ ciclo e de ensino secundário ${ }^{2}$ revelam. Deste modo, procuramos contribuir para uma discussão em torno da pertinência de um quadro teórico sobre o conhecimento estatístico para ensinar, visando o desenvolvimento do pensamento estatístico do aluno, na formação inicial.

\section{Conhecimento estatístico para ensinar}

Vários investigadores têm argumentado a favor da especificidade da Estatística, embora integrada nos currículos de Matemática, dadas as reconhecidas diferenças entre o pensamento estatístico e o raciocínio matemático (GROTH, 2013). Assim, Burgess (2009) propõe um quadro teórico para examinar o conhecimento profissional do professor que tem em conta as necessidades específicas do ensino e aprendizagem da Estatística.

\footnotetext{
${ }^{2}$ Em Portugal, o 3. ${ }^{\circ}$ ciclo do ensino básico diz respeito ao período do 7. ${ }^{\circ}$ ao $9 .^{\circ}$ anos (12-14 anos) e o ensino secundário do $10 .^{\circ}$ ao $12 .^{\circ}$ anos (15-17 anos).
} 
O quadro proposto parte das dimensões de conhecimento matemático para ensinar de Ball, Thames e Phelps (2008), que consideram dois tipos de conhecimento do conteúdo (CC) - conhecimento comum do conteúdo e conhecimento especializado do conteúdo - e dois tipos de conhecimento pedagógico do conteúdo (CPC) conhecimento do conteúdo e dos alunos e conhecimento do conteúdo para ensinar.

$\mathrm{Na}$ matriz que Burgess (2009) propõe para analisar o conhecimento estatístico para ensinar, o conhecimento comum do conteúdo (CCC) refere-se a um tipo de conhecimento comum às profissões que utilizam a Estatística. $\mathrm{O}$ conhecimento especializado do conteúdo (CEC) tem a ver com a capacidade de analisar a adequabilidade das produções do aluno às situações, particularmente se este fez alguma coisa de um modo não expectável (BURGESS, 2009). Inclui a capacidade de justificar os processos e representações utilizadas, como por exemplo, a escolha da medida mais apropriada para um dado conjunto de dados e de compreender a razão dos erros dos alunos, do ponto de vista dos conhecimentos estatísticos. O conhecimento do conteúdo e dos alunos (CCA) combina o conhecimento dos alunos com o conhecimento sobre estatística, permitindo ao professor antecipar o que os alunos pensam sobre um determinado aspeto de um conteúdo, as dificuldades que podem sentir e as suas motivações. Esta dimensão relaciona-se, também, com o conhecimento do professor acerca do pensamento matemático dos alunos, incluindo o conhecimento das conceções adequadas ou erróneas que possam apresentar. Finalmente, o conhecimento do conteúdo para ensinar (CCE) concilia o conhecimento estatístico com as metodologias adequadas para ensinar cada tópico, de modo a promover a aprendizagem dos alunos. Refere-se também à capacidade de selecionar tarefas apropriadas aos objetivos definidos e de as sequenciar.

Há, no entanto, que ressalvar a existência de uma certa sobreposição e interdependência entre estas dimensões, não podendo estas ser encaradas como estanques, na medida em que, por exemplo, o conhecimento especializado e o conhecimento do conteúdo para ensinar (CCE) e conhecimento especializado do conteúdo (CEC) se relacionam com a capacidade de tornar o conteúdo compreensível aos alunos (GROTH, 2013).

Neste quadro, as dimensões do conhecimento descritas (BURGESS, 2009) são, ainda, cruzadas com as componentes do pensamento estatístico de Wild e Pfannkuch (1999) que inclui, no que diz respeito aos tipos de pensamento: 
- o reconhecimento da necessidade de dados - em vez de se apoiar em outro tipo de evidências e perceber que quanto mais dados se tiver melhores conclusões podem ser retiradas;

- a transnumeração - ser capaz de representar os dados de várias formas de modo a ganhar maior significado dos dados;

- a consideração da variação - que influencia a realização de julgamentos a partir dos dados e envolve procura e descrição de parâmetros na variação e tentar compreendê-los em relação ao contexto;

- o raciocínio com modelos - desde os mais simples (como gráficos ou tabelas) até aos mais complexos, permitindo encontrar padrões e sumariar dados de formas múltiplas e usar 'modelos' para raciocinar sobre o problema; e,

- a integração da estatística e contexto - fazer ligações entre os dois, considerando o contexto do problema e como este se articula com o conhecimento estatístico.

Juntamente com estes tipos de pensamento fundamentais existem outros mais gerais que podem ser considerados parte da resolução de problemas, como seja o ciclo investigativo (problema, plano, dados, análise e conclusões) e o ciclo interrogativo (gerar, procurar, interpretar, criticar e julgar) (WILD; PFANNKUCH, 1999).

\section{Contexto e métodos}

O estudo que apresentamos foca-se no conhecimento estatístico para ensinar que futuros professores do $3 .^{\circ}$ ciclo e do ensino secundário evidenciam, nas suas reflexões escritas, e no final de um módulo de uma disciplina de Didática da Matemática lecionada pelas autoras. De um total de 30 aulas da disciplina, de 2,5 horas, seis foram dedicadas ao módulo temático de ensino e aprendizagem da Estatística e Probabilidades.

Neste módulo procurou-se proporcionar aos futuros professores (FP) oportunidades para desenvolverem um conhecimento sobre como ensinar investigações estatísticas, através da realização de uma investigação ao longo de duas aulas. Estes formularam questões de natureza estatística e planearam a recolha de dados. Após a análise de dados, realizada a pares em período extra-letivo, usando uma folha de cálculo, os FP apresentaram as suas explorações e discutiram em grande grupo a escolha apropriada de representações e medidas estatísticas para converter dados na informação necessária para responder às suas questões iniciais e realizar inferências com base na 
interpretação dos resultados obtidos. Foram igualmente foco de debate, as principais orientações didáticas para a exploração de investigações em aula.

Ao terminar este módulo, os FP desenvolveram um trabalho de análise de um caso constituído por episódios de aula do ensino secundário, sobre diferentes fases de uma investigação estatística (retirados de SHAUGHNESSY; CHANCE; KRANENDONK, 2009). A análise de casos de aula é um método de ensino usado na formação que se tem mostrado promissor para preparar os professores na tomada de decisões pedagógicas em ambientes complexos de sala de aula (GROTH; XU, 2011), e que nos pareceu poder proporcionar uma reflexão sobre o processo investigativo vivenciado pelos $\mathrm{FP}$ em sala de aula e, simultaneamente, colocar em evidência o seu conhecimento sobre o ensino deste tema.

Os episódios analisados pelos FP dizem respeito a uma investigação que envolve a análise de dados de censos da população de três países, em que os alunos usam uma variedade de representações dos dados das populações e algumas medidas estatísticas, e focam-se na descoberta e explicação de padrões nos dados e nas diferenças ou semelhanças entre as distribuições das idades da população dos três países. O objetivo do questionamento do professor, nas discussões relatadas nestes episódios, é levar os alunos a desenvolver o raciocínio estatístico, interpretando dados no processo de exploração e a aperceberem-se da constante necessidade de articulação entre dados e contexto.

Os $10 \mathrm{FP}$ da turma realizaram uma reflexão escrita indiviual ou a pares tendo por base a análise que fizeram desses episódios de aula. As reflexões escritas dos FP foram analisadas de forma descritiva e interpretativa, tendo como referência o quadro conceptual de Burgess (2009), em três dimensões do conhecimento do professor - CEC, CCA e CCE - e, simultaneamente, em relação a cada uma das categorias do pensamento estatístico. A partir da análise das reflexões, foi construído um perfil para cada futuro professor ou par de professores que, depois de agregados, produziram um perfil global para a turma (Quadro 1) (HENRIQUES; OLIVEIRA, 2013). Na secção seguinte ilustramos, através de excertos das suas reflexões, o conhecimento estatístico para ensinar evidenciado pelos FP.

\section{Conhecimento estatístico para ensinar dos futuros professores}

No Quadro 1 estão resumidos os aspetos do conhecimento estatístico para ensinar, dos FP $(n=8)$, em relação ao pensamento estatístico. O perfil dos FP mostra 
que a maioria das dimensões do conhecimento do professor em relação às várias componentes do pensamento estatístico está presente nas suas reflexões. No entanto, associada a cada uma das células existe, frequentemente, uma diversidade de conhecimentos pertinentes para o pensamento estatístico. Assim, o facto de uma célula assinalar a presença de conhecimento em relação a uma categoria do pensamento estatístico, não significa que os FP possuam um conhecimento completo de todos os seus aspetos. Da mesma forma, uma célula vazia não significa que os FP não possuam conhecimento relativo a essa dimensão do pensamento estatístico, mas apenas que não o evidenciaram de forma espontânea nas suas reflexões.

Quadro 1: Perfil do conhecimento estatístico para ensinar dos futuros professores $(n=8)$

\begin{tabular}{|c|c|c|c|c|}
\hline & \multirow{2}{*}{$\frac{\mathrm{CC}}{\mathrm{CEC}}$} & \multicolumn{2}{|c|}{$\mathrm{CPC}$} \\
\hline & & & $\mathrm{CCA}$ & $\mathrm{CCE}$ \\
\hline \multirow{5}{*}{ 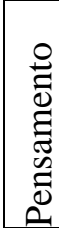 } & Necessidade dos dados & 3 & - & 4 \\
\hline & Transnumeração & 8 & 6 & 7 \\
\hline & Variação & - & 3 & - \\
\hline & Raciocínio com modelos & 8 & 7 & 8 \\
\hline & Integração da estatística e do contexto & 7 & 2 & 6 \\
\hline \multicolumn{2}{|r|}{ Ciclo investigativo } & 4 & 4 & 5 \\
\hline \multicolumn{2}{|c|}{ Ciclo interrogativo } & 5 & - & 4 \\
\hline
\end{tabular}

Conhecimento especializado do conteúdo. Esta dimensão do conhecimento está bastante presente nas reflexões dos FP, em relação à maioria das componentes do pensamento estatístico, estando ausente na componente da variação.

A realização de uma investigação requer, com frequência, a compreensão dos dados que são necessários recolher para responder a uma questão ou problema inicial a ser resolvido. O CEC - necessidade dos dados, que permite reconhecer e avaliar a necessidade dos dados (e a sua recolha intencional) para apoiar a tomada de decisões, está presente em poucas reflexões dos FP, sendo evidenciado apenas quando estes salientam passagens do episódio de aula onde, através das ações do professor, reconhecem que os alunos não compreendem a necessidade de mais dados para se chegar a melhores conclusões: "O professor alerta para que certas conclusões só poderiam ser tiradas com mais informação, referente a outros anos" (FP8).

Conforme evidenciado no Quadro 1, todos os FP manifestam CEC transnumeração nas suas reflexões quando avaliam se as medidas e as representações estatísticas e as suas conexões são usadas de forma apropriada pelos alunos. Este conhecimento inclui, ainda, a capacidade de explicar e justificar quando é que certas 
medidas e representações particulares são mais adequadas que outras para sumariar e facilitar a compreensão de um determinado conjunto de dados, como evidenciado na afirmação seguinte: “As tabelas são utilizadas para organizar os dados e apresentá-los de maneira mais simples. Os gráficos permitem uma melhor visualização e uma análise mais detalhada dos dados apresentados" (FP3).

Em todas as reflexões, os FP evidenciam CEC - raciocínio com modelos quando reconhecem que os modelos usados pelos alunos permitiram dar sentido aos dados e raciocinar corretamente sobre eles. Por exemplo, um FP considera que: "Com a introdução dos histogramas (...) foi fácil para os alunos descobrir a relação entre os histogramas apresentados, para um país como o Quénia onde os dados evidenciavam uma distribuição enviesada, pela sua forma acentuadamente assimétrica (...) e para os restantes países uma distribuição com vários picos ou modas" (FP3).

O CEC - integração da estatística e contexto também surge com frequência nas suas reflexões, mostrando que os FP reconhecem a importância de articular o conhecimento do contexto da situação a investigar com o conhecimento estatístico relacionado com os seus dados. Neste sentido, são capazes de identificar algumas situações no episódio de aula em que os alunos formulam conjeturas com base no que as estatísticas revelam e no contexto, e criticar outras situações em que isso não acontece.

Neste estudo, metade dos FP identifica e caracteriza as fases de uma investigação estatística e avalia o trabalho desenvolvido pelos alunos no episódio de aula em cada uma delas, em particular a correção das questões formuladas e a adequação dos métodos de recolha de dados, evidenciando CEC - ciclo investigativo. A este propósito um FP refere-se às duas fases iniciais:

Na primeira etapa terá que haver cuidado com as questões a colocar e verificar se têm natureza estatística. A segunda, que envolve a recolha de dados, obriga a que exista um plano bem definido com técnicas de recolha de dados apropriadas. (FP1)

O CEC - ciclo interrogativo é evidenciado em cerca de metade das reflexões dos FP, quando reconhecem que a forma como os alunos lidam com os dados é útil para a interpretação posterior dos resultados e para a resposta às questões a investigar. Por exemplo, um FP comenta o envolvimento dos alunos no ciclo interrogativo desta forma: 
resultados, incentivando-se [os alunos a] exprimir as conclusões referentes aos dados e as possíveis generalizações para além dos dados. (FP3)

Conhecimento do conteúdo para ensinar. Este conhecimento é necessário para estruturar o ensino, de modo a encorajar o pensamento estatístico dos alunos. Tal como a anterior, esta dimensão só não está evidenciada nas reflexões dos FP em relação à componente do pensamento estatístico - variação.

Cerca de metade dos FP evidencia CCE - necessidade dos dados, nas suas reflexões, quando defende, por exemplo, que "Deve discutir-se com os alunos a informação que é necessária para tomar uma decisão válida ou tirar uma conclusão fundamentada" (FP1), reconhecendo a importância das discussões em grande grupo para promover a compreensão sobre a necessidade dos dados.

O CCE - transnumeração foi identificado em quase todas as reflexões dos FP e manifesta-se quando estes expressam concordância ou criticam a abordagem usada, considerando que a mesma é adequada ou limita o desenvolvimento da capacidade de transnumeração dos alunos, como evidenciado no excerto seguinte:

Com a elaboração das colunas com percentagens, os alunos começaram a ver com mais clareza as diferenças nas classes etárias (...) [e] a comparar grupos (mais velhos e mais novos). O professor conseguiu desta forma que o aluno pensasse numa forma alternativa de representar a informação. (FP8)

Em outros casos sugerem inclusivamente o uso de abordagens diferentes: " $\mathrm{O}$ alargamento da versatilidade das representações estudadas ajuda a desenvolver o espirito crítico dos alunos, através da discussão de qual das representações dos dados é mais reveladora das características de uma população" (FP6).

Todos os FP expressam CCE - raciocínio com modelos ao reconhecerem que a abordagem adotada pelo professor, quando apresenta em paralelo os diagramas de extremos e quartis relativos a três populações que estavam a ser comparadas, permite o desenvolvimento do raciocínio dos alunos, como mostra o excerto seguinte:

Este encadeamento entre diferentes representações e a crescente complexidade em termos de análise é um bom exemplo de como se pode ajudar os alunos a evoluir nos seus raciocínios. (...) [A] evolução das tabelas para os gráficos e diagramas, permite aos alunos formularem questões mais complexas. (FP2) 
Em vários casos também defendem e justificam o uso da tecnologia para facilitar a construção de representações gráficas e o cálculo de medidas, permitindo que os alunos se foquem nos raciocínios e também "Potenciando a discussão de ideias, a tomada de decisão" (FP3).

O CCE - integração da estatística e contexto é fundamental para utilizar estratégias de ensino adequadas, que levem os alunos a reconhecer a relevância do conhecimento contextual na investigação a desenvolver, e para as avaliar. Neste sentido, vários FP evidenciam este conhecimento nas suas reflexões quando reconhecem, por exemplo, que o professor pretende com esta tarefa que os alunos:

Façam a ligação entre os dados e possíveis factos verídicos e fenómenos sociológicos que os próprios possam conhecer, de forma a compreenderem melhor o que aqueles valores significam (...), nomeadamente, com fenómenos que possam justificar os dados em questão. (FP6)

Reconhecer estratégias de ensino capazes de encorajar os alunos a pensar sobre as fases de uma investigação e a articulação entre elas é uma das componentes do $C C E$ ciclo investigativo. Cerca de metade dos FP evidencia esta dimensão do conhecimento quando, por exemplo, refere como iria propor esta tarefa aos alunos: "começaria por formular um problema; incentivar os alunos a recolherem dados reais a partir do site dos censos; promover a organização e o tratamento dos dados (...) e pediria aos alunos para irem retirando as suas conclusões, justificando-as" (FP5).

O CCE - ciclo interrogativo é evidenciado por metade dos FP quando comentam as estratégias que o professor dos episódios usa para lidar com a tendência dos alunos de limitarem o foco da investigação sobre os dados, ignorando uma vasta gama de outras possibilidades. Por exemplo, referem que:

Com perguntas como: "como descreveriam esta distribuição?", (...) "que mais se pode sumariar sobre a população?", "O que poderá explicar essas diferenças?", o professor pretende que os alunos se questionem, façam conjeturas e discutam a validade das ideias (...) para poderem tirar algumas conclusões sobre a população. (FP6)

Conhecimento do conteúdo e dos alunos. Esta dimensão do conhecimento é a que está menos presente nas reflexões dos FP, sendo que as componentes do pensamento estatístico necessidade dos dados e ciclo interrogativo não foram evidenciadas. 
Os FP evidenciam CCA - transnumeração quando antecipam dificuldades e conceções erradas dos alunos na utilização de diversas representações e na seleção da mais adequada para representar os dados:

O professor (...) com os gráficos, pediu para [os] fazer corresponder aos países sem dar indicações de como ler a informação neles contida. Teria sido (...) de esperar que os alunos manifestassem dificuldades em lê-los, principalmente numa representação gráfica como o diagrama de extremos e quartis que é pouco intuitiva" (FP7)

O CCA - variação inclui a capacidade de prever as dificuldades que os alunos enfrentam na compreensão da variação dos dados e como lidam com tarefas que envolvem este conceito. Este conhecimento, pouco contemplado nas reflexões dos FP, é evidenciado quando referem, por exemplo: "Com um tão grande volume de dados é possível que os alunos tenham alguma dificuldade, nomeadamente na procura de regularidade entre os dados e a identificação de diferenças no sentido de descrever (...) a [sua] variabilidade" (FP1).

Quase todos os FP são capazes de antecipar ou reconhecer as dificuldades dos alunos na utilização de diversos modelos, incluindo tecnologia, para raciocinar sobre os dados. Mostram, assim, CCA - raciocínio com modelos, afirmando:

A leitura dos diagramas de extremos e quartis já não foi tão imediata, pois os alunos precisaram de raciocinar e fazer uso do facto das três amostras terem medidas de localização distintas. Isto era esperado visto que este tipo de diagrama não pertence ao currículo dos anos anteriores. (FP4)

O CCA - integração da estatística e contexto está pouco evidenciado nas reflexões dos FP. No entanto, este é mobilizado no comentário seguinte, quando o FP reconhece que o tema da investigação proposta dificulta a integração do conhecimento contextual no conhecimento estatístico: "Para os alunos do nosso exercício, as questões sociais vieram ao de cima. Mas como é que se pode levar os alunos a interessarem-se (...) [por este tema] se essa sensibilidade não existir?" (FP8).

Metade dos FP evidencia nas suas reflexões CCA - ciclo investigativo. Alguns afirmam-se favoravelmente surpreendidos com o trabalho desenvolvido pelos alunos no episódio de aula mas identificam, igualmente, dificuldades esperadas, mostrando que estão cientes dos aspetos do ciclo investigativo que são problemáticos ou desafiantes: 
As principais dificuldades que podem ocorrer são exatamente na formulação das perguntas - as quais irão definir toda a investigação. As perguntas são relevantes? Têm natureza estatística? Podem [ainda] surgir dificuldades em articular a definição dos dados a recolher com as perguntas elaboradas. Que tipo de dados recolher, o que se espera obter da recolha dos dados? (FP8).

\section{Conclusões}

Através da análise das reflexões escritas dos FP em torno dos episódios de aula, foi possível identificar elementos importantes do quadro de Burgess (2009) sobre o conhecimento do professor para ensinar investigações estatísticas.

No que diz respeito à dimensão do CEC, a generalidade das componentes está bastante presente, evidenciando que os FP são capazes de avaliar as produções dos alunos em termos do conhecimento estatístico envolvido. Salienta-se, relativamente ao pensamento estatístico, que a grande maioria dos FP identifica aspetos relacionados com a transnumeração, o raciocínio com modelos e a integração da estatística e do contexto, o que mostra que está a desenvolver um conhecimento estatístico que não se restringe ao domínio de fórmulas e procedimentos. Assim, os FP evidenciam ser capazes de avaliar as medidas e representações estatísticas que os alunos usam e, também, como estes atribuem sentido aos dados e raciocinam sobre estes, e articulam o conhecimento estatístico e o conhecimento do contexto da situação. Pelo contrário, a componente variação não foi identificada nas suas reflexões, o que pode indiciar, por um lado, que este conceito é novo para eles e, por isso, não estão suficientemente despertos para a sua importância no desenvolvimento do pensamento dos alunos e, por outro lado, que as características dos episódios de aula analisados limitam a mobilização desse conhecimento, uma vez que, nas situações apresentadas, os dados com que os alunos lidam são referentes a populações e não a amostras. Cerca de metade dos FP identifica aspetos do CEC ligadas ao ciclo investigativo, caracterizando as fases de uma investigação estatística e debruçando-se sobre a correcção das questões formuladas e a adequação dos métodos de recolha de dados. Também em relação ao ciclo interrogativo, cerca de metade dos FP evidencia ter desenvolvido algum conhecimento sobre como a interpretação dos resultados e a resposta às questões do estudo dependem do tratamento dos dados.

Relativamente ao CCE e, atendendo à natureza da tarefa utilizada neste estudo, esta dimensão manifesta-se quando os FP são capazes de interpretar as ações do professor dos episódios de aula, evidenciando conhecer várias estratégias de ensino e 
saber justificar a sua adequabilidade para promover a aprendizagem dos alunos. No que diz respeito à promoção do pensamento estatístico dos alunos, a quase totalidade dos FP mostra conhecer e conseguir analisar abordagens que favorecem ou condicionam a capacidade de transnumeração dos alunos, o raciocínio com modelos, reconhecendo em alguns casos o papel da tecnologia na construção de representações gráficas, e a articulação entre conhecimento estatístico e contextual na realização de uma investigação. Apenas a componente variação não está presente nas suas reflexões, o que pode estar relacionado os aspetos referidos a propósito do CEC, assim como com a falta de experiência de lecionação deste aspeto. Os FP manifestam também algum conhecimento relativamente ao modo como promover a realização de investigações (ciclo investigativo) e identificam estratégias, nomeadamente de questionamento, que podem contribuir para os alunos interpretarem os resultados (ciclo interrogativo). $\mathrm{O}$ CCA é a dimensão que tem menor expressão nas reflexões dos FP, o que é natural dado o reduzido contacto que tiveram, até ao momento da sua formação, com alunos em aula, em particular, a realizar investigações estatísticas. Nesta dimensão não se evidenciam as componentes do pensamento estatístico, necessidade dos dados e ciclo interrogativo. No entanto, os FP são capazes de identificar desafios e reconhecer as principais dificuldades que os alunos dos episódios de aula enfrentam, sobretudo em relação às componentes do pensamento que lhes são mais familiares (Transnumeração e Raciocínio com modelos).

Os resultados deste estudo estão naturalmente condicionados pelas características da tarefa proposta, dado que esta não orientava para uma análise específica de cada uma das componentes, o que pode justificar algumas omissões nas reflexões dos FP. Há a salientar que, por vezes, os FP fazem afirmações que se relacionam com aspetos do modelo do conhecimento adotado mas que são demasiado gerais, mostrando-se ainda pouco eficazes para apoiar o raciocínio dos alunos. Apesar das limitações da tarefa proposta, o conhecimento estatístico para ensinar que esta fez emergir reforça as potencialidades da análise de casos de aula na formação inicial de professores (GROTH e XU, 2011). No entanto, dado que a maioria dos FP não experimentou, enquanto alunos, a realização de investigações estatísticas, é fundamental que na sua formação possa experimentar essa atividade, tal como sucedeu com os participantes deste estudo, de uma forma que promova o seu CCC em articulação com o seu CPC (BURGESS, 2009). 
Este estudo permite evidenciar a adequabilidade do modelo conceptual no contexto da formação inicial de professores, embora este tenha sido originalmente construído para descrever o conhecimento estatístico para ensinar de professores em serviço, e ainda que reconhecendo, por vezes, a dificuldade em separar as diferentes componentes (GROTH, 2013), pela interdependência que as caracteriza. Este quadro, pela sua especificidade, pode constituir-se como referência para a construção e proposta de situações a explorar no domínio da Didática da Estatística. Pode, ainda, permitir ao formador analisar como estão os FP a desenvolver o seu conhecimento estatístico para ensinar e que aspetos necessitam aprofundar, desejavelmente em articulação com a prática de ensino supervisionada.

Agradecimento. Estudo realizado no âmbito do Projeto DSL - Developing statistical literacy: Student learning and teacher education, apoiado pela FCT-Fundação para a Ciência e a Tecnologia (contrato PTDC/CPE-CED/117933/2010).

\section{Referências}

BALL, D. L.; THAMES, M. H.; PHELPS, G. Content knowledge for teaching: What makes it special? Journal of Teacher Education, Thousand Oaks, CA, v. 59, n. 5, p. 389-407, Nov./Dec. 2008.

BURGESS, T. A. Teacher knowledge and statistics: What types of knowledge are used in the primary classroom? The Montana Mathematics Enthusiast, Missoula, MT, v. 6, n. 1-2, p. 3-24, Jan. 2009.

CAMPOS, T.; CAZORLA, I.; KATAOKA, V. Statistics School Curricula in Brazil. In BATANERO C.; BURRILL, G.; Reading C. (Ed.), Teaching statistics in school mathematics. Challenges for teaching and teacher education. Dordrecht, The Netherlands: Springer, 2011. p. 5-8.

FRANKLIN, C. et al. A curriculum framework for K-12 statistics education: GAISE report. American Statistical Association, 2005. Disponível em: www.amstat.org/education/gaise/. Acesso em: 13 jun. 2010.

GROTH, R. E. Characterizing key developmental understandings and pedagogically powerful ideas within a statistical knowledge for teaching framework. Mathematical Thinking and Learning, Mahwah, NJ, v. 15, n. 2, p. 121-145, Apr. 2013.

GROTH, R. E.; XU, S. Preparing teachers through case analyses. In BATANERO C.; BURRILL, G.; Reading C. (Ed.). Teaching statistics in school mathematics. 
Challenges for teaching and teacher education. Dordrecht, The Netherlands: Springer, 2011. p. 371-382.

HEATON, R. M.; MICKELSON, W. T. The learning and teaching of statistical investigation in teaching and teacher education. Journal of Mathematics Teacher Education, Dordrecht, The Netherlands, v. 5, n. 1, p. 35-59, Mar. 2002.

HENRIQUES, A. C.; OLIVEIRA, H. Prospective teacher's statistical knowledge for teaching when analyzing classroom episodes. In Proceedings of the $37^{\text {th }}$ Conference of the International Group for the Psychology of mathematics Education, Kiel: PME, 2013. v. 3, p. 41-48.

NEWTON, J.; DIETIKER, L.; HORVATH, A. Statistics Education in the United States: Statistical Reasoning and the Statistical Process. In BATANERO C.; BURRILL, G.; READING C. (Ed.). Teaching statistics in school mathematics. Challenges for teaching and teacher education. Dordrecht, The Netherlands: Springer, 2011. p. 9-13.

SHAUGHNESSY, J. M.; CHANCE, B.; KRANENDONK, H. Focus in high school mathematics: Reasoning and Sense Making in Statistics and Probability. Reston, VA: NCTM, 2009.

WILD, C. J.; PFANNKUCH, M. Statistical thinking in empirical enquiry. International Statistical Review, The Hague, The Netherlands, v. 67, n. 3, p. 223-265, Dec. 1999. 